\title{
Expansion of epigenetic alterations in EFEMP1 promoter predicts malignant formation in pancreatobiliary intraductal papillary mucinous neoplasms
}

\author{
Kazuhiro Yoshida $^{1}$ Takeshi Nagasaka ${ }^{1}(1) \cdot$ Yuzo Umeda $^{1} \cdot$ Takehiro Tanaka $^{2}$ • \\ Keisuke Kimura $^{1} \cdot$ Fumitaka Taniguchi $^{1} \cdot$ Tomokazu Fuji $^{1} \cdot$ Kunitoshi Shigeyasu $^{1}$

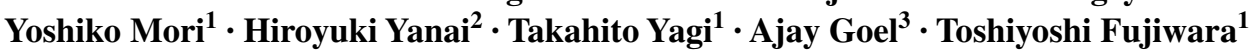

Received: 12 February 2016 / Accepted: 11 April 2016 / Published online: 19 April 2016

(c) The Author(s) 2016. This article is published with open access at Springerlink.com

\begin{abstract}
Purpose Although limited understanding exists for the presence of specific genetic mutations and aberrantly methylated genes in pancreatobiliary intraductal papillary mucinous neoplasms (IPMNs), the fundamental understanding of the dynamics of methylation expansion across $\mathrm{CpG}$ dinucleotides in specific gene promoters during carcinogenesis remains unexplored. Expansion of DNA methylation in some gene promoter regions, such as EFEMP1, one of the fibulin family, with tumor progression has been reported in several malignancies. We hypothesized that DNA hypermethylation in EFEMP1 promoter would expand with the tumor grade of IPMN.

Methods A sample of 65 IPMNs and 30 normal pancreatic tissues was analyzed. IPMNs were divided into the following three subsets according to pathological
\end{abstract}

Electronic supplementary material The online version of this article (doi:10.1007/s00432-016-2164-x) contains supplementary material, which is available to authorized users.

Takeshi Nagasaka

takeshin@cc.okayama-u.ac.jp

1 Department of Gastroenterological Surgery, Okayama University Graduate School of Medicine, Dentistry and Pharmaceutical Sciences, 2-5-1 Shikata-cho, Kita-ku, Okayama City, Okayama 700-8558, Japan

2 Department of Pathology, Okayama University Graduate School of Medicine, Dentistry and Pharmaceutical Sciences, Okayama City, Okayama 700-8558, Japan

3 Center for Gastrointestinal Cancer Research, Center for Epigenetics, Cancer Prevention and Cancer Genomics, Baylor Research Institute and Charles A Sammons Cancer Center, Baylor University Medical Center, Dallas, TX 75246, USA findings: 31 with low-grade dysplasia (low grade), 11 with high-grade dysplasia (high grade), and 23 with associated invasive carcinoma (invasive $\mathrm{Ca}$ ). Mutations in the KRAS or GNAS genes were analyzed by Sanger sequencing, and methylation status of two discrete regions within the EFEMP1 promoter, namely region 1 and region 2, was analyzed by bisulfite sequencing and fluorescent high-sensitive assay for bisulfite DNA (Hi-SA). Expression status of EFEMP1 was investigated by immunohistochemistry (IHC).

Results KRAS mutations were detected in 39, 55, and $70 \%$ of low-grade, high-grade, and invasive $\mathrm{Ca}$, respectively. GNAS mutations were observed in 32,55 , and $22 \%$ of low-grade, high-grade, and invasive $\mathrm{Ca}$, respectively. The methylation of individual regions (region 1 or 2) in the EFEMP1 promoter was observed in 84, 91, and $87 \%$ of low-grade, high-grade, and invasive $\mathrm{Ca}$, respectively. However, simultaneous methylation of both regions (extensive methylation) was exclusively detected in $35 \%$ of invasive $\mathrm{Ca}(p=0.001)$ and five of eight IPMNs $(63 \%)$ with extensive methylation, whereas 20 of $57(35.1 \%)$ tumors of unmethylation or partial methylation of the EFEMP1 promoter region showed weak staining EFEMP1 in extracellular matrix $(p=0.422)$. In addition, extensive EFEMPI methylation was particularly present in malignant tumors without GNAS mutations and associated with disease-free survival of patients with IPMNs $(p<0.0001)$.

Conclusions Extensive methylation of the EFEMP1 gene promoter can discriminate invasive from benign IPMNs with superior accuracy owing to their stepwise accumulation of tumor progression.

Keywords Mucinous neoplasms · Methylation · Epigenetics $\cdot$ EFEMP1 $\cdot$ Invasive carcinoma $\cdot$ Dysplasia 


\section{Introduction}

Pancreatic intraductal papillary mucinous neoplasms (IPMNs) are precursor lesions characterized by an atypical degree of intraductal proliferation of neoplastic mucinous cells arising in the pancreatic duct (Das et al. 2013; Matthaei et al. 2012; Tanaka et al. 2012; Wasif et al. 2010). Histologically, IPMNs may progress from low-grade to high-grade dysplasia and finally to an invasive carcinoma (Das et al. 2013; Farrell and Brugge 2002; Salvia et al. 2004), canonical to the adenoma-carcinoma sequence in colorectal cancer and pancreatic ductal adenocarcinoma (PDAC) (Wasif et al. 2010). While IPMNs with invasive carcinoma have a poor 5-year survival ratio of 33-43\%, patients with resected IPMNs without any invasive cancer features generally have a better 5-year survival ratio of 77-94 \% (Chari et al. 2002; Das et al. 2013; Farrell and Brugge 2002; Maire et al. 2002; Raimondo et al. 2002; Sohn et al. 2004), highlighting the importance of efficient diagnosis of IPMNs with invasive carcinoma. Therefore, preoperative identification of dysplastic IPMNs is challenging even with a multimodality approach including radiographic imaging, endoscopic ultrasound-guided fineneedle aspiration (EUSFNA), cytological examination, and tumor markers (Schoedel et al. 2006). This problem has prompted the development of other analytical tools, including genomic biomarkers that can predict IPMNs at a high risk of developing dysplasia with malignant potential (Schoedel et al. 2006).

Applying molecular techniques to evaluate surgical and cytological specimens is evolving in conjunction with our understanding of the IPMN molecular makeup (Schoedel et al. 2006). Studies aimed at characterizing genetic profiles in IPMNs have identified activating mutations of KRAS and GNAS oncogenes and inactivating mutations in RNF43, $C D K N 2 A / p 16$, and TP53 tumor suppressor genes (Amato et al. 2014; Cooper et al. 2013; Dal Molin et al. 2013; Furukawa et al. 2011; Kanda et al. 2013; Komatsu et al. 2014; Schonleben et al. 2007, 2008; Sessa et al. 1994). Collectively, these studies underscored the importance of genetic alterations in IPMN progression; however, the prevalence of such genetic events generally occurs at lower frequencies than in PDAC (Adsay 2002; Cooper et al. 2013; Kanda et al. 2013; Sato and Goggins 2006).

Similar to other cancers, epigenetic alterations, such as promoter hypermethylation of tumor suppressor genes, are considered a critical process in IPMN development (Sato and Goggins 2006). Results suggest a gradual expansion of methylation across $\mathrm{CpG}$ islands in MGMT, RASSF2, and $S F R P 2$ promoters during colorectal cancer progression and highlighted their potential role as biomarkers for diagnosis and disease prediction for specific cancer types (Nagasaka et al. 2008, 2009; Takeda et al. 2011). In this study, we evaluated the methylation status of the epidermal growth factor-containing fibulin-like extracellular matrix protein 1 gene (EFEMPI, alternative annotation is FIbulin3), a member of the fibulin family of extracellular matrix (ECM) proteins. EFEMP1 is involved in malignant transformation through modulation of cell proliferation, angiogenesis, and invasion in a tissue-dependent manner (Kobayashi et al. 2007; Sadr-Nabavi et al. 2009; Wang et al. 2010, 2012; Yang et al. 2013; Yue et al. 2007; Zhu et al. 2014), and alterations in this gene expression have often been linked to aberrant DNA methylation (Nomoto et al. 2010; Sadr-Nabavi et al. 2009; Wang et al. 2010, 2012; Yang et al. 2013; Yue et al. 2007; Zhu et al. 2014). Moreover, recent studies have reported an association between a reduction in protein expression and EFEMP1 methylation expansion in breast and lung cancers using immunohistochemistry and sequencing approaches (Chen et al. 2014; Sadr-Nabavi et al. 2009). For these reasons, the presence or absence of methylation and gradual expansion of specific gene promoter methylation may help diagnose and differentiate invasive carcinoma from normal adjacent tissues and dysplastic lesions.

To systematically test this hypothesis, we first analyzed mutations in the KRAS and GNAS genes to confirm the genetic background of IPMNs. Next, to determine whether extensive methylation of candidate genes may serve as a predictive alteration for malignant IPMNs, we performed a comprehensive investigation of the methylation status of EFEMP1 promoter and examined EFEMP1 expression status by IHC.

\section{Materials and methods}

\section{Samples and tumor classifications}

Nine tissues from non-necrotic areas of PDAC were frozen immediately at $-80{ }^{\circ} \mathrm{C}$, and DNA was extracted from the tissues using QIAamp DNA mini kits (Qiagen, Valencia, CA, USA). DNAs of 21 tissues from non-necrotic areas of PDAC were macrodissected from formalin-fixed, paraffinembedded (FFPE) specimens. DNAs of IPMNs were macrodissected from 65 patients who underwent surgical resection and who were pathologically diagnosed with IPMNs or invasive IPMNs. All samples were collected from the Okayama University Hospital, Okayama, Japan, between January 2001 and December 2012. Institutional review board approval was granted by the Ethics Committee of the Okayama University, and written informed consent was obtained from all patients to use their tissues for research. The medical records of the patients were retrospectively explored and matched with clinical and pathological data. We defined IPMN classification based on the International Consensus Guidelines from 2012 as follows: MD-IPMNs were 
characterized by segmental or diffused dilation of the main pancreatic duct by $>5 \mathrm{~mm}$ in the absence of other causes of obstruction; BD-IPMNs comprised pancreatic cysts of $>5 \mathrm{~mm}$ in diameter communicating with the main pancreatic duct; finally, mixed-type lesions were those lesions that simultaneously met the criteria of both MD-IPMNs and BDIPMNs (Tanaka et al. 2012). In addition, we used a revised terminology to classify IPMNs. Formally, IPMNs are classified according to World Health Organization classification based on pathological findings as follows: IPMNs with lowgrade dysplasia, intermediate-grade dysplasia, high-grade dysplasia (carcinoma in situ) and associated invasive carcinoma. Based on the revised classification criteria proposed in 2015, IPMNs with intermediate-grade dysplasia are combined together with the ones with low-grade dysplasia and are now called low-grade IMPNs. Therefore, we classified IPMNs according to this revised nomenclature.

\section{IPMN patients' characteristics}

Using the criteria mentioned above, our sample of 65 IPMNs was classified as follows: 31 (48 \%), $11(17 \%)$, and 23 (35\%) IPMNs were classified as low- or intermediate-grade dysplasia (low grade), high-grade dysplasia (high grade), and invasive $\mathrm{Ca}$, respectively. To clarify the clinicopathological features of IPMNs, statistical analyses were performed between low-grade and high-grade and invasive Ca (Supplementary Table 1). Within the group of 23 invasive $\mathrm{Ca}$, only one case showed a distant metastasis in the liver at surgical resection (and hence classified as stage IV); the remainder of the invasive Ca cases was categorized as stage I (10 of 23; $44 \%$ ) and stage II (12 of 23; $52 \%$, Supplementary Table 2). Disease-free survival (DFS) and overall survival (OS) of IPMNs were estimated according to their clinicopathological characteristics (Supplementary Fig. 1). The median time of the follow-up period of 65 IPMNs after surgical resection was 48 months (range 8-108 months). The lesions categorized as invasive $\mathrm{Ca}$ were also classified by the tumor node metastasis classification system (Adsay et al. 2010; Kim et al. 2008).

\section{Direct sequencing of KRAS and GNAS mutations in IPMNS tissues}

KRAS mutations in codons 12 and 13 were determined by the method previously described (Nagasaka et al. 2008, 2009; Takeda et al. 2011). GNAS mutations in codon 201 were analyzed by direct sequencing using GNAS primers (Supplementary Fig. 2 and Supplementary Table 3).

\section{Analysis of DNA methylation}

DNA was subjected to sodium bisulfite modification using the EZ DNA Methylation Kit (ZYMO Research,
Irvine, CA). As shown in Fig. 1, methylation status of the EFEMP1 gene promoter was studied at various regions in previous studies (Kobayashi et al. 2007; Nomoto et al. 2010; Sadr-Nabavi et al. 2009; Wang et al. 2010, 2012; Yang et al. 2013; Yue et al. 2007; Zhu et al. 2014). In this study, we searched CTCF biding sites by CTCFBSDB 2.0 (http://insulatordb.uthsc.edu/) in promoter region of the EFEMP1 gene and found a CTCF biding site 'TGACATCTGTTGGG,' called as the EMBL_M1 motifs (Schmidt et al. 2012). CTCF, also known as CCCTC-binding factor, is a transcription factor involved in many cellular processes, including transcriptional regulation, insulator activity, V(D)J recombination, and regulation of chromatin architecture (Chaumeil and Skok 2012; Phillips and Corces 2009). Interestingly, this biding site was between the two regions which were analyzed by previous studies as mentioned above. For the reason, we divided the EFEMPI gene promoter into two regions (regions 1 and 2, Fig. 1), which were located as dividing line on the CTCF biding site, and analyzed by a fluorescence high-sensitive assay (HiSA) using bisulfite-modified DNA template as previously described (Nagasaka et al. 2009). The sense and antisense nonspecific primers and internal methylation-specific primers with enhanced sensitivity for polymerase chain reaction (PCR) amplification have been described previously (Nagasaka et al. 2009) and are shown in Supplementary Table 3. PCR products digested with HhaI (New England BioLabs, Massachusetts, USA) were loaded simultaneously onto an ABI 310R or 31000 Genetic Analyzer (Applied Biosystems, California, USA). Signals from individual PCR products were distinguished by the unique fluorescent PCR signal from each target and their fragment length, and the data were analyzed using GeneMapper software version 4.0 (Applied Biosystems, California, USA). In this study, the percentages of methylated HhaI sites were calculated by determining the ratios between the HhaI-cleaved PCR product and the total amount of PCR product in each loci, and methylation positivity was defined as a proportion of $>1.0 \%$ of methylated HhaI sites. Direct sequencing of the two regions in the EFEMPI promoter was performed by PCR products obtained from bisulfite DNA extracted from normal pancreatic tissues and IPMNs. Primer sequences are shown in Supplementary Table 3.

\section{Immunohistochemical analysis}

EFEMP1 localization was performed by immunohistochemical (IHC). A sample of 65 IPMNs was available for IHC staining for EFEMP1 protein expression analysis. Staining was carried out manually with FFPE tissues. Thin $(5 \mu \mathrm{m})$ sections of representative blocks were deparaffinized and dehydrated using gradient solvents. Following antigen retrieval in the citrate buffer $(\mathrm{pH} 6.0)$, endogenous 

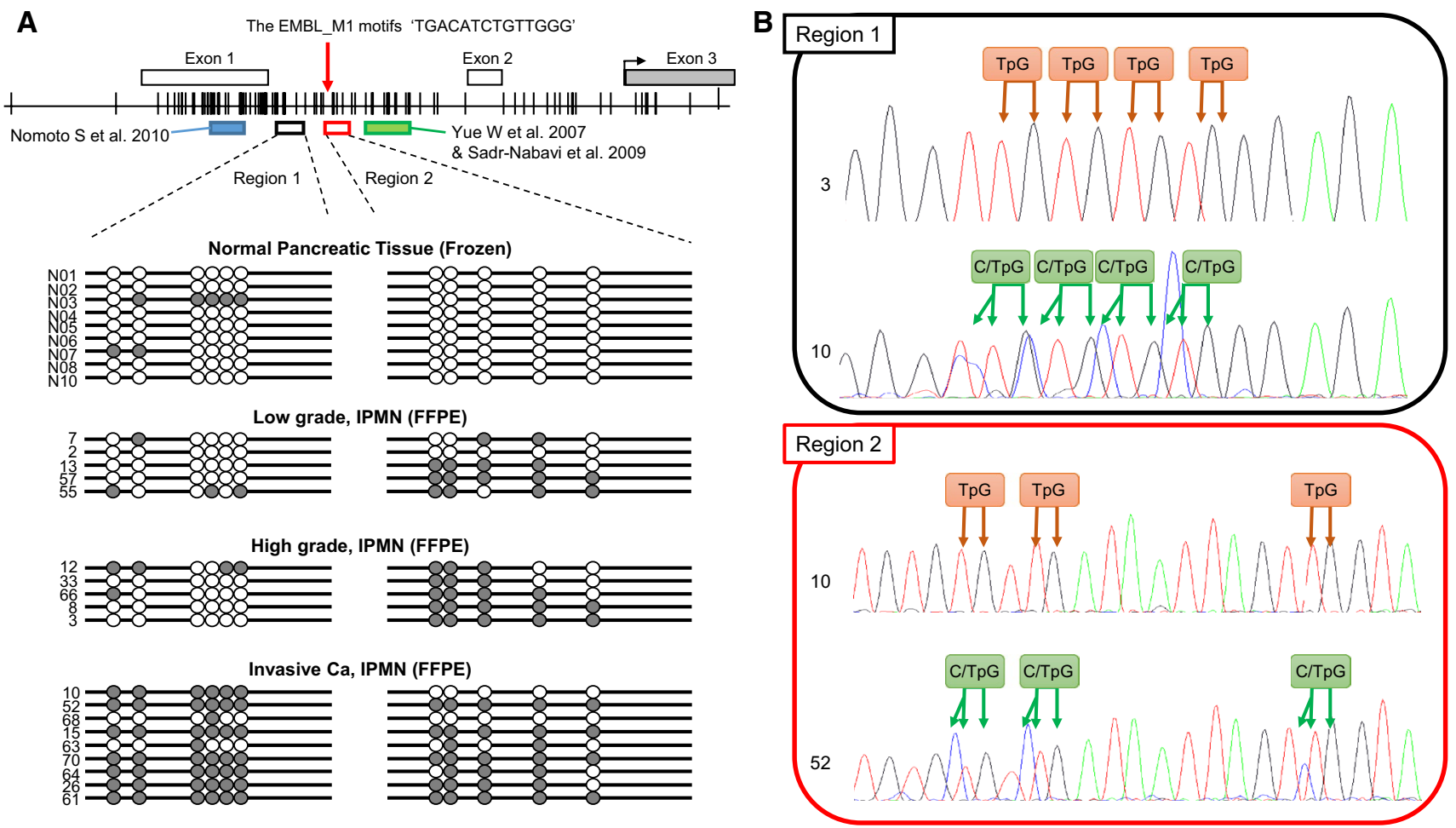

Fig. 1 Bisulfite sequencing of the discrete EFEMP1 gene promoter regions. a Schematic representation of the location of discrete EFEMP1 gene promoter regions and the result of bisulfite sequencing. The white and gray boxes denote untranslated and translated exon in the EFEMP1 gene, respectively. The red allow indicates the location of the EMBL_M1 motifs 'TGACATCTGTTGGG', a candidate of CTCF biding site. The black arrow indicates the transcriptional starting site. The blue box indicates the regions of which methylation status was analyzed by Nomoto et al. (2010). The green

peroxidase was blocked with $3 \% \mathrm{H}_{2} \mathrm{O}_{2}$. Thereafter, slides were incubated overnight in the presence of a purified mouse anti-human EFEMP1 monoclonal antibody (sc33722, Santa Cruz, Dallas, TX, USA; dilution 1:250). Further incubation was carried out with a secondary antibody and the avidin-biotin-peroxidase complex (Vector Laboratories, Burlingame, CA, USA) and then incubated with biotinyl-tyramide followed by streptavidin-peroxidase. Diaminobenzidine was used as a chromogen and hematoxylin as a nuclear counterstain.

EFEMP1 was detectable in normal pancreatic tissue; weak staining was detected in islets of Langerhans, whereas intense staining was observed in the peripheral nerve fiber. IHC results were interpreted by pathologists blinded to the corresponding clinicopathological data. The expression status of EFEMP1 was evaluated by an immunoreactive score (IRS), which was calculated by scoring of the percentage of positive cells and their expression intensities. The percentage of positive ECM staining was rated as described previously and as follows: $1=0-10 \%, 2=11-50 \%$, box indicates the regions of which methylation status was analyzed by Yue et al. (2007) and Sadr-Nabavi et al. (2009). Vertical lines indicate CpG sites; white circles represent unmethylated CpGs; and gray circles represent methylated and unmethylated $\mathrm{CpGs}$ observed by bisulfite direct sequencing. $\mathbf{b}$ Examples of bisulfite sequencing in region 1 and 2. Each $\mathrm{CpG}$ was categorized as unmethylated or methylated CpG. TpG denotes the $\mathrm{CpG}$ site consisting of unmethylated $\mathrm{CpG}$ only. $\mathrm{C} / \mathrm{TpG}$ denotes the $\mathrm{CpG}$ site consisting of both methylated $(\mathrm{CpG})$ and unmethylated $\mathrm{CpGs}(\mathrm{TpG})$

$3=51-80 \%$, and $4=81-100 \%$ (Sadr-Nabavi et al. 2009). Staining intensity was scored as follows: $1=$ weak, $2=$ moderate, and $3=$ intensive. All IPMNs were categorized into four subsets by IRS score as follows: $0-1=$ no staining, $2-3=$ weak staining, $4-8=$ moderate staining, and $9-12=$ strong staining (Remmele and Stegner 1987).

\section{Statistical analyses}

All statistical analyses were performed using EZR (Saitama Medical Center, Jichi Medical University), which is a graphical user interface for R (The R Foundation for Statistical Computing, version 2.13.0). First, methylation levels were analyzed as continuous variables. Next, the methylation status was analyzed as a categorical variable (positive, methylation level $>1.0 \%$; negative, methylation level $\leq 1.0 \%$ ), as described previously. Each IPMN specimen was given a numerical score so as to reflect the number of methylated loci. Categorical variables were compared by Fisher's exact test. Differences between continuous 
variables were determined using the Mann-Whitney $U$ test or the Kruskal-Wallis test. Multiple comparisons were performed using the Steel-Dwass test. OS was calculated from the date of surgical resection to the date of death due to IPMNS or last follow-up for censored patients. DFS was calculated from the date of surgical resection to the date of the first documentation of local, regional, or distant relapse, appearance of a second primary lesion by computed tomography and/or magnetic resonance imaging routinely performed per 6 months. OS and DFS were univariately estimate with the Kaplan-Meier method. All $p$ values reported were calculated by two-sided tests, and values $<0.05$ were considered statistically significant.

\section{Results}

\section{KRAS and GNAS mutations in IPMNs}

To evaluate the genetic background in our current cohort, we analyzed mutations in KRAS and GNAS genes as shown in Supplementary Figure 2. KRAS mutations were detected in 33 IPMNs $(51 \%)$, and the spectrum of relative frequencies of individual mutations was 5 (15\% of KRAS mutants), 12 (36\%), and 16 (49 \%) for G12R, G12D, and G12V mutations, respectively (Supplementary Tables 1 and 2). Meanwhile, 19 IPMNs (29\%) harbored GNAS mutations, and the following mutations were primarily found in codon 201: R201H, 5 IPMNs (26 \%); R201C, 13 IPMNs (68 \%); and R201S, 1 IPMNs (5 \%) (Table 2). One R201S was a novel mutation, which had not been previously described for IPMNs. KRAS mutation frequency tended to increase with IPMN grade: 12 of 31 were low grade (39\%), 6 of 11 were high grade $(55 \%)$, and 16 of 23 were invasive $\mathrm{Ca}$ (70 \%, $p=0.084$; low-grade vs. high-grade/invasive Ca). In contrast, GNAS mutations were constantly observed, with 10 of $32(32 \%)$ in low-grade and 12 of $33(36 \%)$ in high-grade and invasive $\mathrm{Ca}$. Concurrent KRAS and GNAS mutations were observed in 11 IPMNs (17\%), KRAS mutation were present only in 22 IPMNs (34 \%), GNAS mutations only in 8 IPMNs (12\%), and wild-type status of both genes was observed in 24 IPMNs (37 \%, Supplementary Fig. 2C).

We assessed DFS and OS according to KRAS or GNAS mutation status. Although KRAS mutations were more frequently observed in invasive $\mathrm{Ca}$, there was no difference in outcome between KRAS mutants and wild type (Supplementary Fig. 2E and F). In contrast, GNAS mutations were less frequently observed in invasive $\mathrm{Ca}$ compared with low and high grade, and there was no difference in outcome between GNAS mutants and wild type (Supplementary Fig. 2G and $\mathrm{H}$ ).

\section{Methylation profiles of EFEMP1 promoter in IPMNs}

We investigated the methylation status of discrete regions in the EFEMP1 promoter in 65 IPMNs and 30 normal pancreatic tissues obtained from PDAC patients. The location of the EFEMPI gene and a panel of representative bisulfite sequencing and fluorescent $\mathrm{Hi}-\mathrm{SA}$ results are depicted in Figs. 1 and 2a, respectively. Methylation status in the discrete regions obtained from fluorescent Hi-SA was analyzed as the categorical variable. Figure $2 \mathrm{~b}$ presents methylation frequency in each discrete region according to pathological features, and Table 1 presents the correlation between the methylation status of EFEMPI and the clinical and pathological features of IPMNs. The region-1 methylation was frequently observed in invasive $\mathrm{Ca}(p=0.0016)$, whereas the region-2 methylation was commonly observed in IPMN (>80 \% of IPMNs) but less frequently in normal pancreatic tissues $(<20 \%)$. Another interesting feature of IPMNs with extensive EFEMP1 methylation was histologic subtype, in which extensive EFEMP1 methylation was frequently observed in pancreatobiliary type lesions (4 of $6 ; 67 \%, p=0.027$ ).

With respect to KRAS/GNAS mutation status, interestingly, IPMNs with methylation of the region-1 never harbored GNAS mutations ( 0 of $10 ; 0 \%, p=0.028$ ), while 7 of 10 IPMNs with methylation of the region-1 harbored KRAS mutations (70\%). While methylation of the region-2 promoter was frequently observed (54 of 65 IPMNs [83\%]), no associations were observed among the frequencies of region- 2 methylation and any of the clinicopathological factors.

Next, we evaluated correlations between the methylation status of EFEMPI promoter and the clinicopathological features of invasive $\mathrm{Ca}$ (Supplementary Table 3). However, methylation status had no association with any of the features explored in invasive $\mathrm{Ca}$.

Finally, we also assessed DFS and OS according to methylation status in the discrete EFEMPI promoter regions. As extensive $E F E M P 1$ methylation was a specific feature of invasive $\mathrm{Ca}$, IMPNs with extensive EFEMPI methylation showed a poor prognosis compared with IMPNs without extensive EFEMP1 methylation (Fig. 2c, d).

\section{Association of EFEMP1 promoter methylation and protein expression}

We investigated EFEMP1 protein expression in 65 IPMN tissues. Representative examples of IHC staining results are shown in Fig. 3a-c. Using these criteria, no IPMNs were categorized as having no staining, whereas 25,38 , and 2 IPMNs were deemed to have weak, moderate, and strong 
A

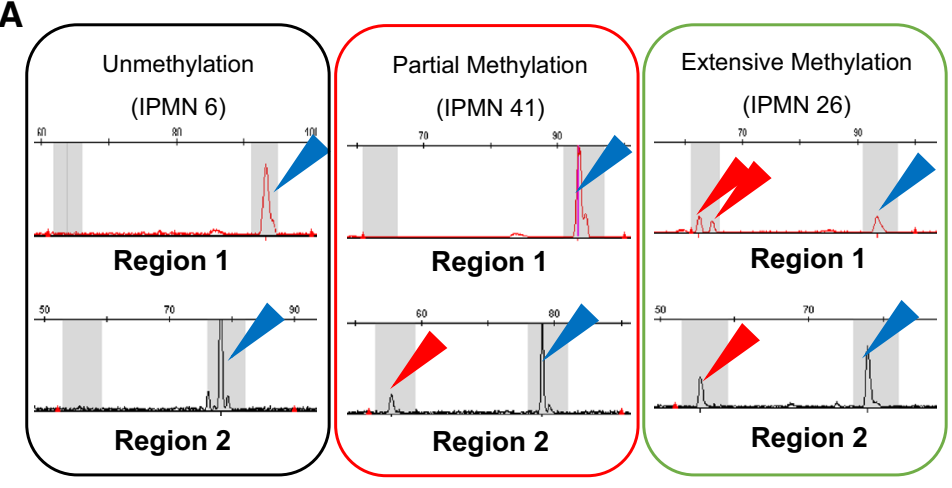

B

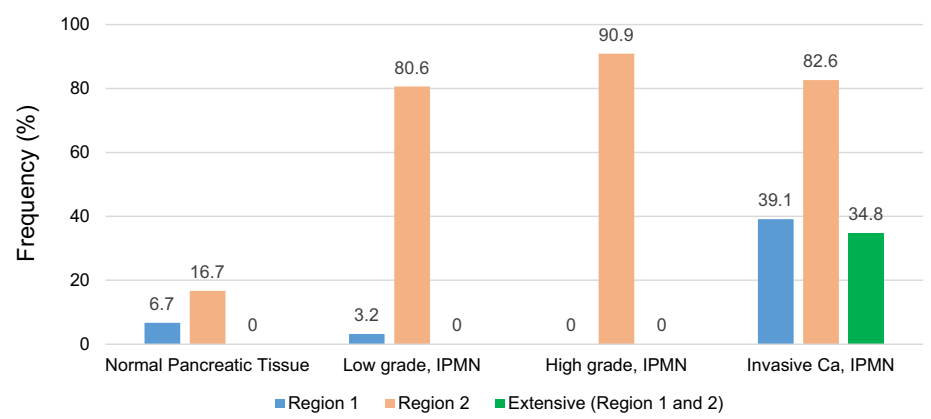

C

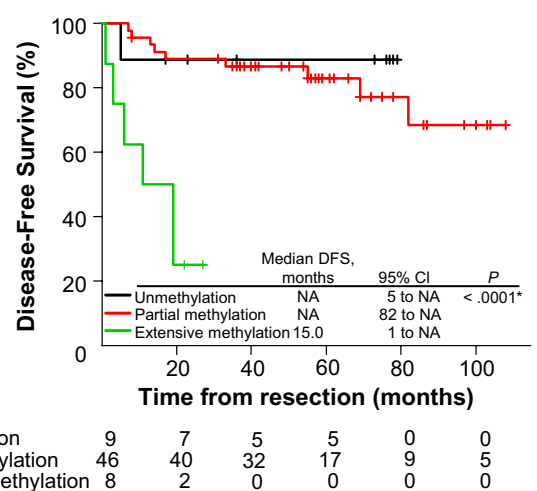

D

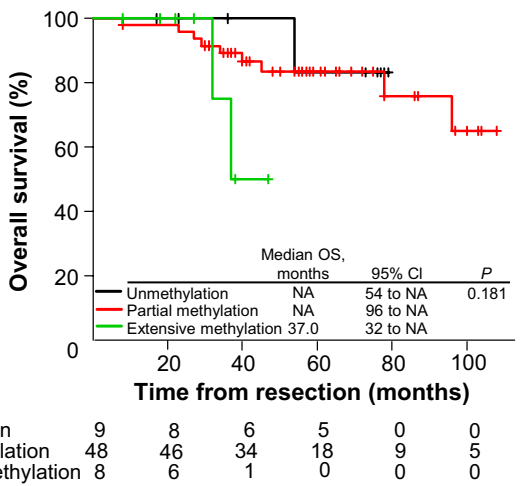

Fig. 2 Methylation analysis of EFEMP1. a Results of EFEMP1 methylation by fluorescent Hi-SA. Blue arrows represent PCR fragments with non-methylation in HhaI sites. Red arrows represent methylated PCR fragments cleaved by HhaI. b Frequencies of
EFEMP1 methylation according to pathological findings. KaplanMeier survival curves for disease-free survival (c), excluding a patient with remaining cancer at the resected margin, and overall survival (d) according to EFEMP1 promoter methylation status staining, respectively. Although five of eight IPMNs (63\%) showed weak staining and extensive methylation of the EFEMP1 promoter region, no significant differences in the frequencies of EFEMP1 protein expression were observed in IPMNs exhibiting partial methylation or non-methylation in the EFEMP1 promoter (Fig. 3d).

\section{Discussion}

We have shown for the first time the biological significance of methylation in discrete promoter regions of EFEMPI gene in tissue specimens obtained from patients with pancreatobiliary IPMNs, playing an important functional role in malignant transformation by modulating cell proliferation, angiogenesis, and invasion in a tissue-dependent context (Kobayashi et al. 2007); it is a common target of promoter hypermethylation in various tumors (Nomoto et al. 2010; Sadr-Nabavi et al. 2009; Wang et al. 2010, 2012; Yang et al. 2013; Yue et al. 2007; Zhu et al. 2014). Aberrant hypermethylation of the EFEMP1 promoter region is a potential biomarker. Sadr et al. reported an association between reduction in protein expression and EFEMPI methylation expansion in breast cancer using sequencing approaches and IHC (Sadr-Nabavi et al. 2009). In this study, we also examined the association between EFEMP1 expression in ECM by IHC and EFEMP 1 methylation profiles. Unfortunately, although $63 \%$ of IPMNs with extensive methylation of the EFEMP1 promoter region showed weak staining in ECM, no significant differences in the frequencies of EFEMP1 protein expression were observed in IPMNs exhibiting partial methylation or non-methylation in the EFEMPI promoter.

Considering the importance of EFEMP1, we hypothesized that gradual expansion of methylation across its promoter during the development of IPMN serves as a biomarker for distinguishing malignant IPMNs from the nonmalignant ones. To systematically test this hypothesis, we first analyzed mutations in the KRAS and GNAS oncogenes to confirm the genetic background of IPMNs. Usually, the methylation pattern in a gene promoter is considered either entirely methylated or non-methylated. However, as demonstrated by bisulfite sequencing, individual $\mathrm{CpG}$ residues within the EFEMP1 promoter were not equally methylated. In this study, region-2 of the EFEMP1 promoter was more frequently methylated than region- 1 , and 


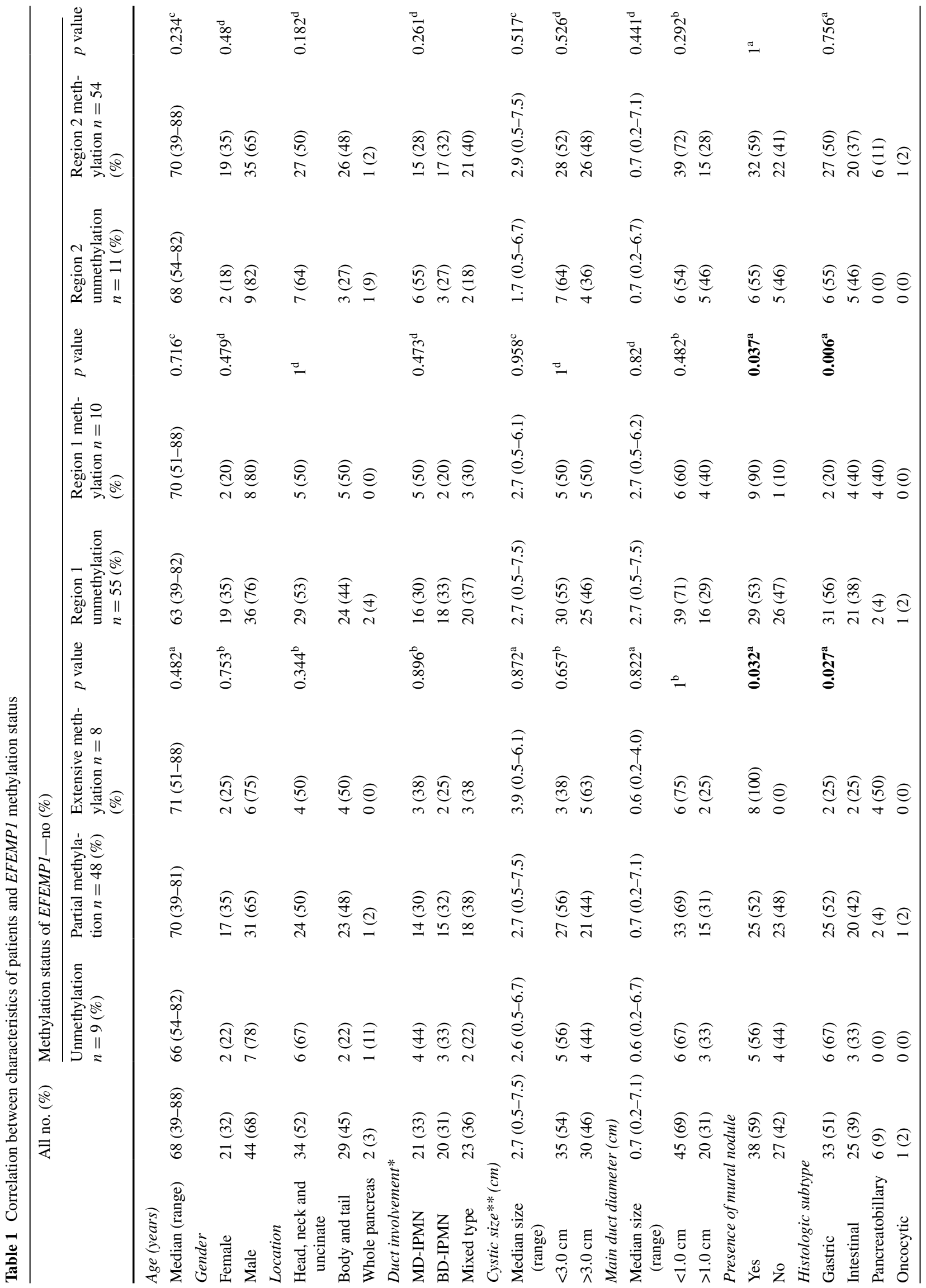




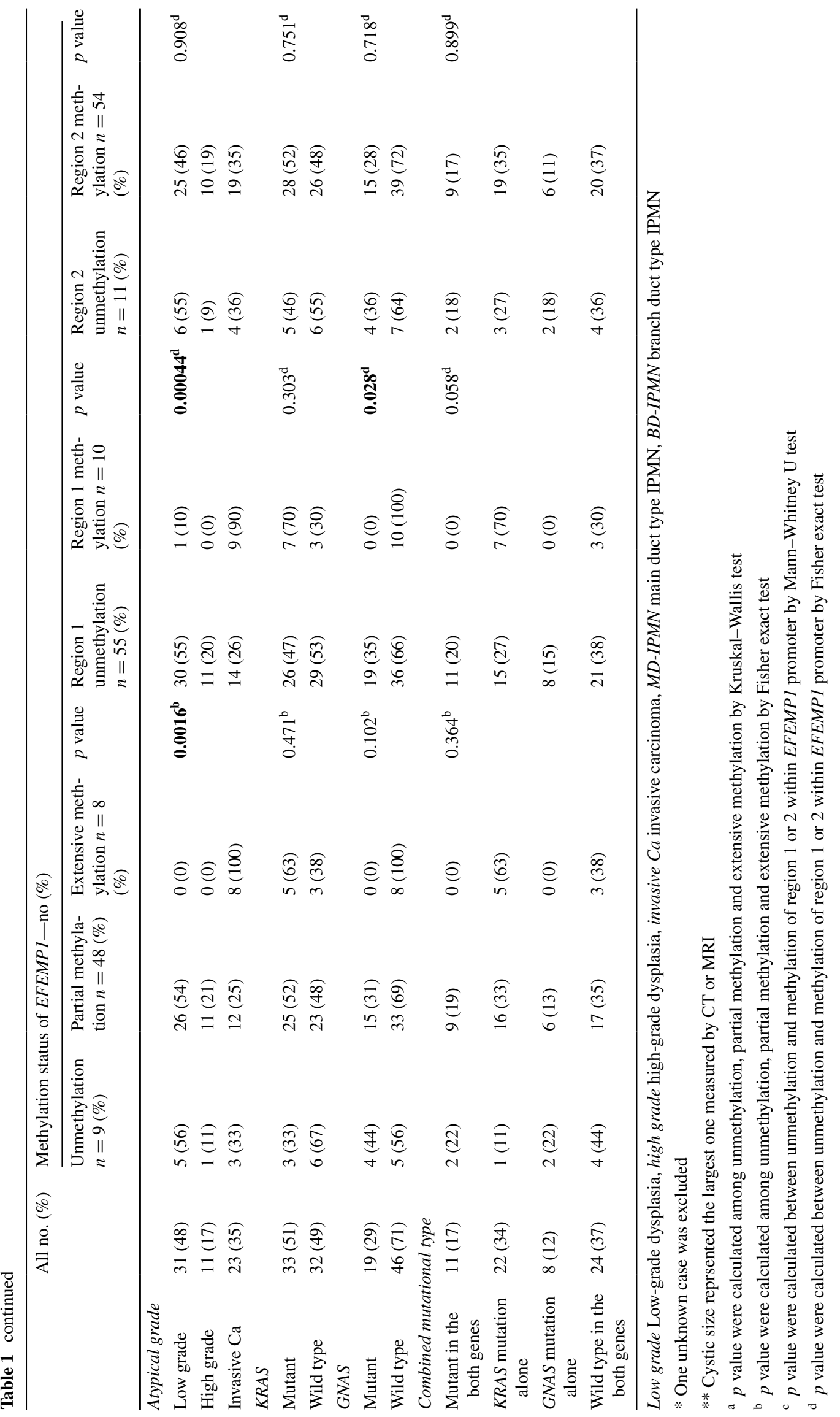




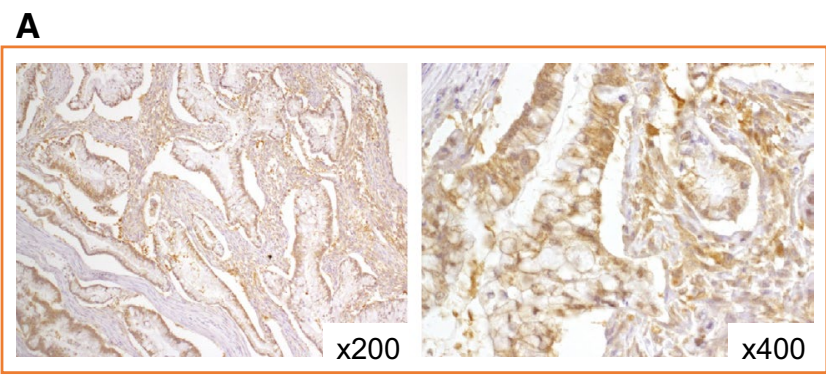

C

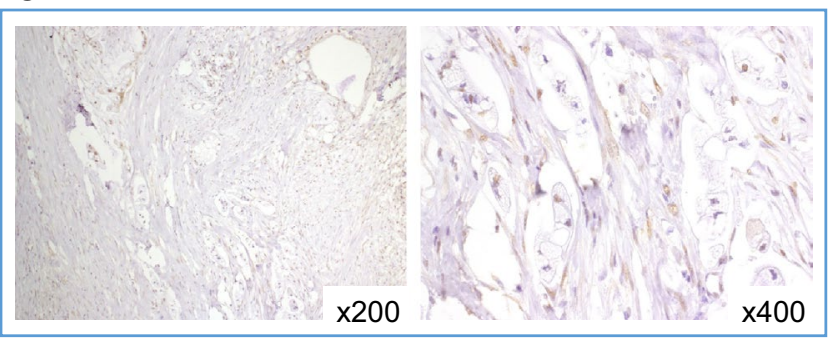

Fig. 3 Expression analysis of EFEMP1. IHC staining of EFEMP1 in intraductal papillary mucinous neoplasms with strong staining (a), moderate staining (b), and weak staining (c). Association between

the aberrant methylation tended to spread from region-2 toward region-1 with IPMN progression. More importantly, none of the normal pancreatic tissues, low- or high-grade IMPNs showed extensive methylation in the EFEMP1 promoter. These characteristics of the EFEMPl methylation pattern in IPMN carcinogenesis appeared similar to those of MGMT, SFRP2, and RASSF2 in the adenoma-carcinoma sequence of colorectal cancer (Nagasaka et al. 2008, 2009; Takeda et al. 2011). Therefore, the presence or absence of methylation and the gradual expansion of methylation of specific gene promoters may help diagnose and differentiate invasive carcinoma from normal adjacent tissues and dysplastic lesions. This fundamental concept of stepwise expansion of DNA methylation across specific gene promoters during the neoplastic progression of IPMNs remains unexplored and is still an active area of investigation.

$K R A S$ and GNAS mutations, the most common genetic mutations observed in IPMNs, occurred in the early stages of disease progression. KRAS and GNAS mutations were also found at codon 12 (a G12D, G12V, or G12R) and codon 201 (an R201H or R201C), respectively. These genetic features are in line with those previously reported in other studies (Amato et al. 2014; Furukawa et al. 2011; Sadr-Nabavi et al. 2009; Wu et al. 2011a, b). This agreement indicates that KRAS mutations at codon 12 or GNAS mutations at codon 201 could play a key role as the driver of carcinogenesis, providing a selective advantage in tumor formation associated with these IPMNs (Parmigiani et al. 2009). However,
B

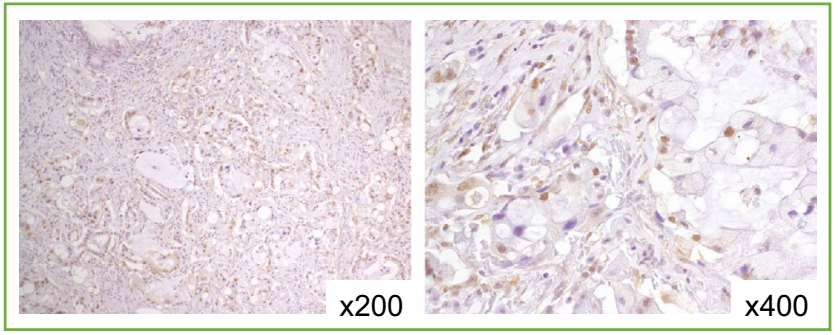

D

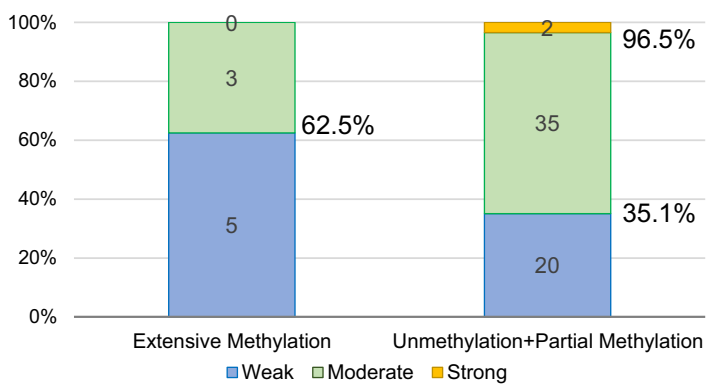

EFEMP1 methylation status and IHC staining (d). EFEMP1, epidermal growth factor-containing fibulin-like extracellular matrix protein 1; IHC immunohistochemical; NA not available

the frequencies of both genetic mutations, especially GNAS mutations, were different among genetic mutational analyses. In the current study, GNAS mutations were observed with similar frequencies in low-grade and invasive $\mathrm{Ca}$. However, Furukawa et al. (2011) reported that GNAS mutations were more common in low-grade lesions. In contrast, Amato et al. and other authors showed that GNAS mutation frequency tended to increase with tumor progression (Amato et al. 2014; Wu et al. 2011a, b). These differences in observations might partly be due to smaller sample size or variations in the detection technologies used in the studies.

To our knowledge, no studies have been published so far demonstrating a correlation between genetic and epigenetic alterations in IPMNs although several previous reports have revealed that there are distinct patterns of genetic mutations in IPMN subtypes (Amato et al. 2014; Chadwick et al. 2009; Cooper et al. 2013; Dal Molin et al. 2013; Fritz et al. 2009; Komatsu et al. 2014; Mino-Kenudson et al. 2011; Mohri et al. 2012). In this study, extensive methylation of EFEMP1 was likely to occur in IPMNs without GNAS mutations (Table 2). This result might reflect the biological behavior of the GNAS gene pathway, which is less aggressive than that of the KRAS gene, and further investigation is needed to evaluate the roles of KRAS and GNAS mutations in IPMN carcinogenesis.

Our study has several limitations. One is the sample size. Another is that, although we examined the correlation between methylation status of our analyzed regions in the 
Table 2 Methylation status of EFEMP1and Genetic profiles of $K R A S$ and GNAS in this cohort

\begin{tabular}{lllllll}
\hline Sample no. Gender & Age & Duct involvement & KRAS & GNAS & EFEMP & \\
\cline { 4 - 5 } & & & & Region 1 Region 2
\end{tabular}

\begin{tabular}{|c|c|c|c|c|c|c|c|}
\hline \multicolumn{8}{|c|}{ Low grade } \\
\hline 14 & $\mathrm{M}$ & 71 & MD & WT & WT & $\mathrm{U}$ & $\mathrm{U}$ \\
\hline 44 & M & 54 & Mixed & WT & WT & $\mathrm{U}$ & $\mathrm{U}$ \\
\hline 47 & M & 58 & MD & WT & WT & $\mathrm{U}$ & $\mathrm{U}$ \\
\hline 46 & $\mathrm{M}$ & 63 & $\mathrm{BD}$ & WT & R201C & $\mathrm{U}$ & $\mathrm{U}$ \\
\hline 7 & $\mathrm{~F}$ & 66 & $\mathrm{BD}$ & WT & WT & $\mathrm{U}$ & $\mathrm{U}$ \\
\hline 22 & $\mathrm{~F}$ & 63 & Mixed & WT & WT & $\mathrm{U}$ & M \\
\hline 50 & $\mathrm{M}$ & 68 & Mixed & WT & WT & $\mathrm{U}$ & M \\
\hline 54 & $\mathrm{~F}$ & 76 & $\mathrm{BD}$ & WT & WT & $\mathrm{U}$ & M \\
\hline 60 & $\mathrm{M}$ & 66 & Mixed & WT & WT & $\mathrm{U}$ & M \\
\hline 65 & $\mathrm{M}$ & 63 & MD & WT & WT & $\mathrm{U}$ & M \\
\hline 39 & $\mathrm{M}$ & 64 & $\mathrm{BD}$ & WT & R201C & $\mathrm{U}$ & M \\
\hline 42 & $\mathrm{M}$ & 59 & MD & G12V & WT & M & $\mathrm{U}$ \\
\hline 56 & $\mathrm{M}$ & 65 & $\mathrm{BD}$ & G12V & R201C & $\mathrm{U}$ & M \\
\hline 17 & $\mathrm{~F}$ & 71 & $\mathrm{BD}$ & WT & WT & $\mathrm{U}$ & M \\
\hline 21 & $\mathrm{M}$ & 67 & $\mathrm{BD}$ & WT & WT & $\mathrm{U}$ & M \\
\hline 31 & $\mathrm{M}$ & 78 & MD & WT & WT & $\mathrm{U}$ & M \\
\hline 35 & $\mathrm{~F}$ & 57 & $\mathrm{BD}$ & WT & WT & $\mathrm{U}$ & M \\
\hline 36 & $\mathrm{~F}$ & 62 & BD & WT & WT & $\mathrm{U}$ & M \\
\hline 2 & $\mathrm{M}$ & 61 & Mixed & WT & WT & $\mathrm{U}$ & M \\
\hline 58 & $\mathrm{M}$ & 60 & $\mathrm{BD}$ & WT & $\mathrm{R} 201 \mathrm{H}$ & $\mathrm{U}$ & M \\
\hline 13 & $\mathrm{~F}$ & 74 & MD & G12R & WT & $\mathrm{U}$ & M \\
\hline 30 & $\mathrm{M}$ & 73 & Mixed & G12D & WT & $\mathrm{U}$ & M \\
\hline 57 & $\mathrm{M}$ & 74 & Mixed & G12R & WT & $\mathrm{U}$ & M \\
\hline 67 & $\mathrm{M}$ & 81 & $\mathrm{BD}$ & G12D & WT & $\mathrm{U}$ & M \\
\hline 55 & $\mathrm{~F}$ & 71 & MD & G12V & R201C & $\mathrm{U}$ & M \\
\hline 41 & M & 50 & $\mathrm{BD}$ & G12D & WT & $\mathrm{U}$ & M \\
\hline 1 & $\mathrm{~F}$ & 65 & Mixed & G12V & $\mathrm{R} 201 \mathrm{H}$ & $\mathrm{U}$ & M \\
\hline 69 & $\mathrm{M}$ & 71 & Mixed & G12D & R201S & $\mathrm{U}$ & M \\
\hline 18 & M & 68 & $\mathrm{BD}$ & WT & R201C & $\mathrm{U}$ & M \\
\hline 25 & $\mathrm{M}$ & 69 & Mixed & G12D & R201C & $\mathrm{U}$ & M \\
\hline 9 & M & 73 & Mixed & G12V & R201C & $\mathrm{U}$ & M \\
\hline \multicolumn{8}{|c|}{ High grade } \\
\hline 12 & $\mathrm{M}$ & 81 & MD & WT & WT & $\mathrm{U}$ & M \\
\hline 16 & M & 72 & MD & WT & WT & $\mathrm{U}$ & M \\
\hline 33 & $\mathrm{~F}$ & 70 & Mixed & G12D & WT & $\mathrm{U}$ & M \\
\hline 43 & M & 75 & Mixed & G12V & $\mathrm{R} 201 \mathrm{H}$ & $\mathrm{U}$ & M \\
\hline 66 & M & 79 & Mixed & WT & R201C & $\mathrm{U}$ & M \\
\hline 19 & $\mathrm{~F}$ & 67 & Mixed & G12R & WT & $\mathrm{U}$ & M \\
\hline 38 & M & 66 & Mixed & G12D & $\mathrm{R} 201 \mathrm{H}$ & $\mathrm{U}$ & $\mathrm{U}$ \\
\hline 8 & M & 73 & Mixed & WT & WT & $\mathrm{U}$ & M \\
\hline 3 & $\mathrm{M}$ & 70 & MD & G12V & WT & $\mathrm{U}$ & M \\
\hline 34 & $\mathrm{~F}$ & 59 & $\mathrm{BD}$ & WT & WT & $\mathrm{U}$ & M \\
\hline 53 & M & 72 & MD & WT & R201C & $\mathrm{U}$ & M \\
\hline \multicolumn{8}{|c|}{ Invasive $\mathrm{Ca}$} \\
\hline 48 & $\mathrm{~F}$ & 66 & Mixed & WT & WT & $\mathrm{U}$ & M \\
\hline 49 & $\mathrm{M}$ & 67 & MD & WT & R201C & $\mathrm{U}$ & $\mathrm{U}$ \\
\hline 10 & M & 73 & MD & G12V & WT & M & $\mathrm{U}$ \\
\hline
\end{tabular}


Table 2 continued

\begin{tabular}{llllllll}
\hline Sample no. & Gender & Age & Duct involvement & KRAS & GNAS & EFEMP & \\
\cline { 6 - 7 } & & & & & & Region 1 & Region 2 \\
\hline 37 & F & 59 & Unknown & G12V & WT & U & M \\
4 & F & 73 & MD & G12V & WT & U & M \\
59 & M & 66 & Mixed & G12V & WT & U & U \\
6 & F & 82 & MD & G12D & R201C & U & U \\
20 & M & 74 & MD & G12D & R201H & U & M \\
32 & M & 56 & BD & WT & WT & U & M \\
11 & M & 74 & MD & WT & R201C & U & M \\
52 & M & 51 & Mixed & G12R & WT & M & M \\
68 & M & 78 & MD & WT & WT & M & M \\
15 & M & 67 & MD & G12D & WT & M & M \\
24 & F & 72 & BD & G12V & WT & U & M \\
51 & M & 57 & MD & G12D & WT & U & M \\
62 & F & 81 & BD & G12V & R201C & U & M \\
63 & M & 75 & BD & WT & WT & M & M \\
70 & M & 57 & Mixed & WT & WT & M & M \\
5 & F & 74 & MD & G12V & WT & U & M \\
40 & M & 39 & Mixed & G12V & WT & M & M \\
64 & M & 63 & BD & G12D & WT & M & M \\
26 & F & 79 & MD & G12V & WT & M & M \\
61 & M & 88 & Mixed & G12R & WT & M & M \\
\hline
\end{tabular}

$M$ methylation, $U$ unmethylation, MD, MD-IPMN; BD, BD-IPMN; Mixed, Mixed-IPMN

EFEMP promoter and its expression status by IHC, there was no strong correlation between them; hence, further investigation is needed. Beyond the limitations, we demonstrated that the extensive methylation in the EFEMP1 promoter could be a useful predictive marker for invasive IPMNs and could serve as a possible means to noninvasively screen for invasive IPMNs using DNA obtained from EUSFNA, pancreatic juice, and fecal samples.

Acknowledgments The authors would like to thank Mr. Toru Nakai, Mrs. Tae Yamanishi, and Mr. Akihiro Nyuya for technical assistance and Enago (www.enago.jp) for English language editing.

\begin{abstract}
Authors' contributions KY extracted DNA, performed methylation and genetic analyses, and drafted the manuscript. TN assisted with data interpretation, designed the project, secured the funding, and drafted the manuscript. YU and TY provided patient samples and clinicopathological data. TT and HY performed IHC and pathological investigations. KK and FT assisted with KRAS and GNAS mutation analysis and provided clinical information. TomF, YM, and SK assisted with IHC staining and summarized clinicopathological data. AG assisted with data interpretation and revised the manuscript. TosF provided patient samples and clinicopathological data, assisted with data interpretation, and revised the manuscript. All authors have read and approved the final manuscript.
\end{abstract}

\section{Compliance with ethical standards}

Conflict of interest All the authors declare that they have no conflict of interest.
Funding This study was funded by KAKENHI (20590572, 25860409, 26462016, and 15H03034).

Ethical approval All procedures were performed in accordance with the ethical standards of the institutional and/or national research committee and with the 1964 Declaration of Helsinki and its later amendments or comparable ethical standards.

Informed consent Informed consent was obtained from all individual participants included in the study.

Open Access This article is distributed under the terms of the Creative Commons Attribution 4.0 International License (http://creativecommons.org/licenses/by/4.0/), which permits unrestricted use, distribution, and reproduction in any medium, provided you give appropriate credit to the original author(s) and the source, provide a link to the Creative Commons license, and indicate if changes were made.

\section{References}

Adsay NV (2002) Intraductal papillary mucinous neoplasms of the pancreas: pathology and molecular genetics. J Gastrointest Surg 6:656-659

Adsay NV, Fukushima N, Furuawa T et al (2010) Intraductal neoplasm of the pancreas. In: Bosman FT, Carneiro F, Hruban RH, Theise ND (eds) WHO classification of tumors of digestive system. WHO press, Lyon, pp 304-313

Amato E et al (2014) Targeted next-generation sequencing of cancer genes dissects the molecular profiles of intraductal papillary 
neoplasms of the pancreas. J Pathol 233:217-227. doi:10.1002/ path.4344

Chadwick B, Willmore-Payne C, Tripp S, Layfield LJ, Hirschowitz S, Holden J (2009) Histologic, immunohistochemical, and molecular classification of 52 IPMNs of the pancreas. Appl Immunohistochem Mol Morphol 17:31-39. doi:10.1097/ PAI.0b013e 31817c02c6

Chari ST et al (2002) Study of recurrence after surgical resection of intraductal papillary mucinous neoplasm of the pancreas. Gastroenterology 123:1500-1507

Chaumeil J, Skok JA (2012) The role of CTCF in regulating V(D)J recombination. Curr Opin Immunol 24:153-159. doi:10.1016/j. coi.2012.01.003

Chen X, Meng J, Yue W, Yu J, Yang J, Yao Z, Zhang L (2014) Fibulin-3 suppresses Wnt/beta-catenin signaling and lung cancer invasion. Carcinogenesis 35:1707-1716. doi:10.1093/carcin/ bgu023

Cooper CL, O'Toole SA, Kench JG (2013) Classification, morphology and molecular pathology of premalignant lesions of the pancreas. Pathology 45:286-304. doi:10.1097/PAT.0b013e32835f2205

Dal Molin M et al (2013) Clinicopathological correlates of activating GNAS mutations in intraductal papillary mucinous neoplasm (IPMN) of the pancreas. Ann Surg Oncol 20:3802-3808. doi:10.1245/s10434-013-3096-1

Das KK et al (2013) mAb Das-1 is specific for high-risk and malignant intraductal papillary mucinous neoplasm (IPMN). Gut 63:1626-1634. doi:10.1136/gutjnl-2013-306219

Farrell JJ, Brugge WR (2002) Intraductal papillary mucinous tumor of the pancreas. Gastrointest Endosc 55:701-714

Fritz S et al (2009) Global genomic analysis of intraductal papillary mucinous neoplasms of the pancreas reveals significant molecular differences compared to ductal adenocarcinoma. Ann Surg 249:440-447. doi:10.1097/SLA.0b013e31819a6e16

Furukawa $\mathrm{T}$ et al (2011) Whole-exome sequencing uncovers frequent GNAS mutations in intraductal papillary mucinous neoplasms of the pancreas. Sci Rep 1:161. doi:10.1038/srep00161

Kanda M et al (2013) Mutant TP53 in duodenal samples of pancreatic juice from patients with pancreatic cancer or high-grade dysplasia. Clin Gastroenterol Hepatol 11(719-730):e5. doi:10.1016/j. $\operatorname{cgh} .2012 .11 .016$

Kim SC et al (2008) Intraductal papillary mucinous neoplasm of the pancreas: clinical characteristics and treatment outcomes of 118 consecutive patients from a single center. J Hepatobiliary Pancreat Surg 15:183-188. doi:10.1007/s00534-007-1231-8

Kobayashi N et al (2007) A comparative analysis of the fibulin protein family. Biochemical characterization, binding interactions, and tissue localization. J Biol Chem 282:11805-11816. doi:10.1074/ jbc.M611029200

Komatsu $\mathrm{H}$ et al (2014) A GNAS mutation found in pancreatic intraductal papillary mucinous neoplasms induces drastic alterations of gene expression profiles with upregulation of mucin genes. PLoS One 9:e87875. doi:10.1371/journal.pone.0087875

Maire $F$ et al (2002) Prognosis of malignant intraductal papillary mucinous tumours of the pancreas after surgical resection. Comparison with pancreatic ductal adenocarcinoma. Gut 51:717-722

Matthaei $\mathrm{H}$ et al (2012) Clinicopathological characteristics and molecular analyses of multifocal intraductal papillary mucinous neoplasms of the pancreas. Ann Surg 255:326-333. doi:10.1097/ SLA.0b013e3182378a18

Mino-Kenudson $\mathrm{M}$ et al (2011) Prognosis of invasive intraductal papillary mucinous neoplasm depends on histological and precursor epithelial subtypes. Gut 60:1712-1720. doi:10.1136/ gut.2010.232272

Mohri D et al (2012) Different subtypes of intraductal papillary mucinous neoplasm in the pancreas have distinct pathways to pancreatic cancer progression. J Gastroenterol 47:203-213. doi:10.1007/s00535-011-0482-y

Nagasaka T et al (2008) Methylation pattern of the O6-methylguanine-DNA methyltransferase gene in colon during progressive colorectal tumorigenesis. Int J Cancer 122:2429-2436. doi:10.1002/ijc. 23398

Nagasaka T et al (2009) Analysis of fecal DNA methylation to detect gastrointestinal neoplasia. J Natl Cancer Inst 101:1244-1258. doi:10.1093/jnci/djp265

Nomoto $S$ et al (2010) Epidermal growth factor-containing fibulinlike extracellular matrix protein 1, EFEMP1, a novel tumorsuppressor gene detected in hepatocellular carcinoma using double combination array analysis. Ann Surg Oncol 17:923-932. doi:10.1245/s10434-009-0790-0

Parmigiani G, Boca S, Lin J, Kinzler KW, Velculescu V, Vogelstein B (2009) Design and analysis issues in genome-wide somatic mutation studies of cancer. Genomics 93:17-21. doi:10.1016/j. ygeno.2008.07.005

Phillips JE, Corces VG (2009) CTCF: master weaver of the genome. Cell 137:1194-1211. doi:10.1016/j.cell.2009.06.001

Raimondo M, Tachibana I, Urrutia R, Burgart LJ, DiMagno EP (2002) Invasive cancer and survival of intraductal papillary mucinous tumors of the pancreas. Am J Gastroenterol 97:25532558. doi:10.1111/j.1572-0241.2002.06022.x

Remmele W, Stegner HE (1987) Recommendation for uniform definition of an immunoreactive score (IRS) for immunohistochemical estrogen receptor detection (ER-ICA) in breast cancer tissue. Pathologe 8:138-140

Sadr-Nabavi A et al (2009) Decreased expression of angiogenesis antagonist EFEMP1 in sporadic breast cancer is caused by aberrant promoter methylation and points to an impact of EFEMP1 as molecular biomarker. Int J Cancer 124:1727-1735. doi:10.1002/ ijc. 24108

Salvia R et al (2004) Main-duct intraductal papillary mucinous neoplasms of the pancreas: clinical predictors of malignancy and long-term survival following resection. Ann Surg 239:678-685 (discussion 685-677)

Sato N, Goggins M (2006) Epigenetic alterations in intraductal papillary mucinous neoplasms of the pancreas. J Hepatobiliary Pancreat Surg 13:280-285. doi:10.1007/s00534-005-1056-2

Schmidt D et al (2012) Waves of retrotransposon expansion remodel genome organization and CTCF binding in multiple mammalian lineages. Cell 148:335-348. doi:10.1016/j.cell.2011.11.058

Schoedel KE, Finkelstein SD, Ohori NP (2006) K-Ras and microsatellite marker analysis of fine-needle aspirates from intraductal papillary mucinous neoplasms of the pancreas. Diagn Cytopathol 34:605-608. doi:10.1002/dc.20511

Schonleben $\mathrm{F}$ et al (2007) BRAF and KRAS gene mutations in intraductal papillary mucinous neoplasm/carcinoma (IPMN/ IPMC) of the pancreas. Cancer Lett 249:242-248. doi:10.1016/j. canlet.2006.09.007

Schonleben F, Qiu W, Remotti HE, Hohenberger W, Su GH (2008) PIK3CA, KRAS, and BRAF mutations in intraductal papillary mucinous neoplasm/carcinoma (IPMN/C) of the pancreas. Langenbecks Arch Surg 393:289-296. doi:10.1007/ s00423-008-0285-7

Sessa $F$ et al (1994) Intraductal papillary-mucinous tumours represent a distinct group of pancreatic neoplasms: an investigation of tumour cell differentiation and K-ras, p53 and c-erbB-2 abnormalities in 26 patients. Virchows Archiv Int $\mathrm{J}$ Pathol 425:357-367

Sohn TA, Yeo CJ, Cameron JL, Hruban RH, Fukushima N, Campbell KA, Lillemoe KD (2004) Intraductal papillary mucinous neoplasms of the pancreas: an updated experience. Ann Surg 239:788-797 (discussion 797-789) 
Takeda M et al (2011) Expansion of CpG methylation in the SFRP2 promoter region during colorectal tumorigenesis. Acta Med Okayama 65:169-177

Tanaka M et al (2012) International consensus guidelines 2012 for the management of IPMN and MCN of the pancreas. Pancreatology 12:183-197. doi:10.1016/j.pan.2012.04.004

Wang R, Zhang YW, Chen LB (2010) Aberrant promoter methylation of FBLN-3 gene and clinicopathological significance in non-small cell lung carcinoma. Lung Cancer 69:239-244. doi:10.1016/j.lungcan.2009.10.009

Wang Z, Yuan X, Jiao N, Zhu H, Zhang Y, Tong J (2012) CDH13 and FLBN3 gene methylation are associated with poor prognosis in colorectal cancer. Pathol Oncol Res POR 18:263-270. doi:10.1007/s12253-011-9437-0

Wasif N, Bentrem DJ, Farrell JJ, Ko CY, Hines OJ, Reber HA, Tomlinson JS (2010) Invasive intraductal papillary mucinous neoplasm versus sporadic pancreatic adenocarcinoma: a stagematched comparison of outcomes. Cancer 116:3369-3377. doi: $10.1002 /$ cncr. 25070

Wu J et al (2011a) Whole-exome sequencing of neoplastic cysts of the pancreas reveals recurrent mutations in components of ubiquitin-dependent pathways. Proc Natl Acad Sci USA 108:21188-21193. doi:10.1073/pnas.1118046108

Wu J et al (2011b) Recurrent GNAS mutations define an unexpected pathway for pancreatic cyst development. Sci Transl Med 3:92ra66. doi:10.1126/scitranslmed.3002543

Yang T et al (2013) Epigenetic inactivation of EFEMP1 is associated with tumor suppressive function in endometrial carcinoma. PLoS One 8:e67458. doi:10.1371/journal.pone.0067458

Yue W et al (2007) Frequent inactivation of RAMP2, EFEMP1 and Dutt1 in lung cancer by promoter hypermethylation. Clin Cancer Res Off J Am Assoc Cancer Res 13:4336-4344. doi:10.1158/1078-0432.CCR-07-0015

Zhu XJ, Liu J, Xu XY, Zhang CD, Dai DQ (2014) Novel tumorsuppressor gene epidermal growth factor-containing fibulin-like extracellular matrix protein 1 is epigenetically silenced and associated with invasion and metastasis in human gastric cancer. Mol Med Rep 9:2283-2292. doi:10.3892/mmr.2014.2135 